\title{
Análisis idiomático de la literatura citada en los artículos científicos del Annual Review of Energy
}

\author{
Felipe Meneses
}

\section{RESUMEN}

Se es tu dia, uti li zan do la téc ni ca bi blio mé tri ca análi sis de ci tas, el idio ma en quees tán ins cri tas 10,211 re fe ren cias de 190 ar tículos del An nual Re view of Energy (Vol. 1, 1976-Vol. 11,1986). Los re sul ta dos re fle jan que los cien tífi cos oc ci den ta les que pu blican en esta fuen te, ci tan es ca sa men te la li te ra tu ra ge ne ra da en el mun do orien tal. S e hace pa ten te el gra ve pro ble ma de la ba rre ra del idioma entre los investigadores que dan a conocer sus escritos en dicha publicación.

\section{SUMMARY}

One studies, uti lizing the bi blio me tric analysis of the quo ta tions, the lan gua ge in whichare written the 10,211 re fe ren ces of the 190 ar ti cles of the An nual Re view of Energy (Vol. 1, 1976, vol. 11, 1986). The re sults re flect what the Wes tern scien tists who publish in this source, ci ting the li te ra tu re ge ne rally in the Eas tern world scar cely at a ll. The se rious pro blem of the lan gua ge ba rriers is made clear among the researchers who know their writings in this publication.

\section{INTRODUCCION}

El presente es tu dio se cir cuns cribe a un análi sis de la li te ra tura citada en el Annual Review of Energy, tomando como variable fundamental el idioma en que están escritas las referencias. Para este propósitoseutilizala téc nicabibliométrica conocida como análisis de citas.

La co lec ción que se es tu dia as cien de a un to tal de once vo lúmenes, del volumen 1, año 1976, al volumen 11, año 1986, con 190 artículos y 10,211 referencias.

Con este trabajo se desea mostrar el grave problema que representa la barrera del idioma entre un grupo de científicos dedicados al estudio de la energía.

\section{NOTAS TEORICAS}

\section{La información científica}

Hoy en día el recurso de mayor importancia para el hombre de ciencia lo representa la información que generan los diver sos cír cu los cien tífi cos y téc ni cos, pro duc to de las in ves tigaciones que se realizan en los ámbitos académicos, gubernamentales y privados. La producción de la información cien tífica y su trans fe ren cia y re cu pe ración opor tu na coad yuvan, de acuerdo con Bergeijk, ' a impulsar:

- el desarrollo científico;

- el progreso económico; y

- el mejoramiento de la sociedad
Des de 1665 , año en que se es ti ma que apa re cie ron las pri meras revistas científicas ${ }^{2}$ (Philosophical Transactions y Journal de Scavans), has ta nues tros días, el prin ci pal so por te documental en que se da a conocer el avance científico y tecnológico es a través de la revista especializada, más específicamente ahora mediante el artículo científico. Este es apoyado por la paten te, el in forme téc ni co y las me morias de congresos.

De acuerdo con Piganiol, ${ }^{4}$ la información científica que presenta las siguientes características es:

- Multi e internacional;

- Internacional;

- Políglota.

A estas características le agregamos una cuarta: aumenta exponencialmente.

El análisis de cada una de estas características nos conducea enfrentarnos, para lograr proporcionar lo que cada científico necesita, a graves problemas tales como:

- barrera del idioma;

- complejidad del conocimiento científico;

- explosión documental, y

- barreras políticas. ${ }^{6}$

Para pro pó si to de este tra bajo so la men te es tu dia re mos el primer problema. 


\section{La barrera lingüística}

La ba rre ra del idio ma es to da vía uno de los prin ci pa les obs tá culos a que se enfrentan los científicos, tecnólogos y profesionales de la documentaciónpara una efectiva transferencia y recuperación de la información.

La ba rre ra lin güís ti ca, como tam bién se le co no ce a di cho obstáculo, es el problema que representa para los científicos de Occidente consultar documentos escritos en idiomas orientales, tales como ruso, japonés, coreano, polaco, entre otros. Aun que cabe acla rar que la ba rre ra del idio ma igual men te está pre sen te en tre los paí ses del mun do oc ci den tal con los idio mas que se hablan dentro de esta parte del planeta.

Según opinión de Bergeijk, ${ }^{1}$ la barrera lingüística ejerce una influencia esencial y creciente sobre la difusión de la información. Estudios realizados por Wood, Anderson, ${ }^{7}$ y Kunicki aprueban este criterio.

Wood fue quien pre ci sa men te, a fi nes de la déca da del se senta, dio la voz de alarma sobre el problema que representaba para los cien tífi cos in gle ses las len guas orien ta les. Este au tor demostró, con los resultados de su investigación, que poco menos del $50 \%$ de la cien cia era publi ca da en idio mas dis tintos al inglés; también dedujo que el ruso era el idioma más im por tan te, des pués del in glés, por la can ti dad y ca li dad de la literatura científica que se publicaba, en ese entonces, en esa lengua.

Años más tarde, a fi nes de la dé ca da del se ten ta para ser más preciso, Kunicki continuó haciendo patente la preocupación del pro ble ma del idio ma cuan do es cri bió los efec tos de la ba rrera de la lengua en su artículo "La barriere linguistique : son importance et son évaluation" y que publicara a principios de los ochenta en Documentaliste.

También se ha hecho evidente cómo afecta la barrera del idioma en el campo de los bibliotecólogos. El estudio titulado "Librarians and the language barrier", escrito por Dickson, ${ }^{10}$ refleja los problemas que se generan en el ámbito bibliotecológico por el poco conocimiento que se tiene sobre otros idio mas y sin te ti za los prin ci pa les es fuer zos que, des de 1948, se han venido haciendo para superar este problema en el te rrenoeducativo. Asi mis mo, manifies ta la gran preocu pación por la es ca sa im por tan cia que se le ha dado para in tro ducir, en las escuelas de bibliotecología, materias tendientes a solucionar el grave problema.

Finalmente, los esfuerzos al querer utilizar una lengua internacionalmente, como el esperanto," no han sido hasta la fecha la mejor solución para superar la barrera del idioma, así como tampoco lo ha sido las recomendaciones de aprender idiomas, por parte del científico, o la publicación de toda la literatura científica en una misma lengua. ${ }^{12}$

De acuer do con lo an te rior, los ex per tos en la ma te ria coin ciden en señalar que el camino más idóneo a seguir es la traducción del documento científico, independientemente de todo el trabajo que esto implica.

\section{La traducción}

Las ta reas des ti na das a re sol ver el proble ma de la tra duc ción del documento científico se han desarrollado tanto en países occidentales como orientales. En tre los pri me ros es tán: Es tados Unidos de Norteamérica, Canadá, Inglaterra, Francia y Es pa ña; por los paí ses orien ta les cabe des ta car a la Unión Soviética y Japón.

El progre so al can za do has ta la fe cha en ma te ria de tra duc ciones, tanto manuales como automatizadas, varía de país a país; sin embargo, los avances no han sido del todo satisfactorios en virtud de los resultados obtenidos.

Lain ter ven ción de paí ses oc ci den ta les y orien ta les en el di seño, estudio y ejecución de proyectos sobre traducción automatizada refleja la gran preocupación de la barrera del idioma y hace evidente que la diversidad de lenguas es el problema más grave para una eficaz transferencia y recuperación de información.

Desde la década del cincuenta, en que se inicia la presentación de sistemas y proyectos sobre la traducción automatizada, hasta hoy día, no se ha logrado resolver el problema que representa el prescindir completamente de toda intervención humana $^{13}$ en lo que res pecta a la tra duc ción de li te ra tu racientífica a través del uso de la computadora.

Actualmente, uno de los principales soportes en materia de traducción está representado por las instituciones que se dedican a realizar y almacenar traducciones en todas las áreas del conocimiento humano, de las que cabe destacar a: el National Translations Center ${ }^{14}$, de los Estados Unidos de Norteamérica; la British Library Division, de Inglaterra, y el Centro Internacional de Traducciones en Holanda, entre otros.

Las actividades que se desarrollan en estos organismos son tan im por tan tes como va ria das. Para tal efec to se re co mien da consultar el estudio de Arnaiz, ${ }^{15}$ quien describe los servicios y publicaciones más sobresalientes de los mismos.

En resumen, la traducción como vía para superar la barrera del idioma es, como se mencionó antes, la más idónea y sobre la cual se trabaja arduamenteendiversos paísesinteresados, ${ }^{16}$ con el objeto de hacer más accesible la información científica.

Para ilustrar el problema planteado se ha hecho el estudio de análisis de citas del Annual Review of Energy.

\section{NOTAS REFERENCIALES}

\section{El Annual Review of Energy}

La información que se produce en los diferentes campos científicos se registra comúnmente en forma de artículo, el cual pasa, junto con otro grupo de documentos similares, a formar un volumen de una revista especializada.

Un ejemplo de este tipo de material es el Annual Review of Energy que, des de que apa re ció el Vol. 1, año 1976, ha ve ni do 
publicando una serie de artículos selectos, inherentes al área energética, con enfoque tanto científico como humanístico.

Lo versátil de su contenido, en cuanto que trata estudios sobre todo tipo de fuentes de energía y te mas afi nes, lo hace ser uno de los documentos de mayor consulta en la Biblioteca del Pro gra ma Uni ver si ta rio de Ener gía y, por tan to, es un material de suma utilidad para el apoyo de las diversas investigaciones que desarrolla la UNAM y otras instituciones del sector energético mexicano.

El Annual Re view of Energy lo publica, durante el mes de octubre, la Annual Review Inc., en Palo Alto, California. Este edi tor tie ne como pro pó si to fun da men tal con tribuir al avan ce de las ciencias.

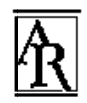

\section{ANNUAL REVIEW OF ENERGY}

VOLUML 11, 1986

\author{
JACK M. HOLLANDER, Editor \\ The Ohis State Unirersity \\ HARYEY BROOKS, Asrociate Ediror \\ Haymand Universily \\ DAVID STERNLIGHT, Associat? Editor \\ Pasaden, Califomia
}

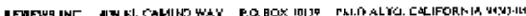

Portada de uno de los volúmenes del An nual Re view of Energy

La em pre sa An nual Re view Inc.ini ció sus ac ti vi da des edi toriales en 1931 con la publicación Annual Review of Biochemistry. Dicha empresa, que desde el principio se propuso como función principal elaborar publicaciones de alta calidad y ofrecerlas a un precio razonable, tiene en su haber los siguientes títulos: ${ }^{17}$

\section{Annual Reviews of:}

Anthropology

Astronomy \& Astrophysics

Biochemistry

Biophysics \& Biophysical Chemistry

Cell Biology

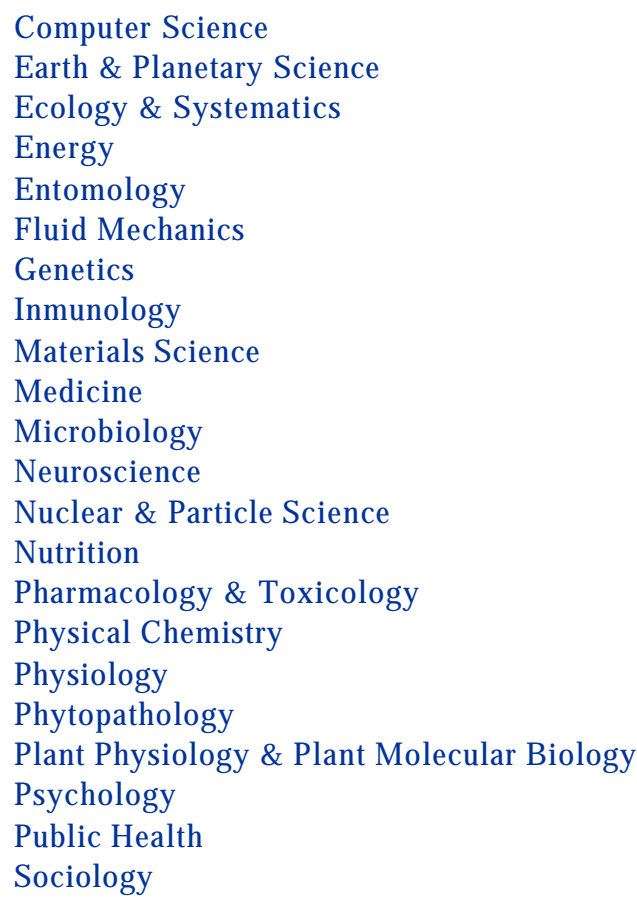

Los volúmenes son organizados por los Comités Editoriales, conformados por personas calificadas, es decir, que han contribuido de manera significativa al desarrollo de cada una de las disciplinas. La Annual Review Inc. es administrada por un Consejo de Directores.

\section{Board of Directors} Membership of June 1, 1988

J. Murray Luck. Founder and Director Emeritus of Annual Reviews Inc.

Professor Emeritus of Chemistry, Stanford University

Joshua Lederberg, Prsident of Annual Reviews, Inc. President, The Rockefeller University

James E. Howell. Vice-President of Annual Reviews Inc. Professor of Ecnomics, Stanford University

Winslow R. Briggs, Director. Carnegie Institution of Washington, Stanford

Sidney Drell, Deputy Director. Stanford Linear Accelerator Center

Eugene Grafield, President. Ins titu te for Scientific In formation

William Kaufmann, President. William Kaufmann, Inc.

D.E. Koshland, Jr. Pro fessor of Bio che mis try. Uni ver sity of California, Berkeley

Gardner Lindzey. Director. Center for Advanced Study in the Behavioral Sciences, Stanford 
William F. Miller, President. SRI International

Harriet A. Zuckerman, Professor of Sociology, Columbia University

Como po de mos ob ser var, los miem bros del Con se jo son profesores universitarios y directivos de institutos de gran prestigio; entre los que podemos identificar al Dr. Eugene Garfield, director del Institute for Scientific Information y autor de varios escritos en el ámbito documental.

De acuerdo con los datos expuestos, se puede afirmar que el Annual Review of Energy es una fuente primaria de información cien tífi ca de ab so lu ta se rie dad y de gran pres ti gio, re conocida tanto en el campo de la energía como en las áreas afines.

\section{OBJETIVOS DEL ESTUDIO}

\section{Objetivo general}

Investigar aspectos cuantitativos del conglemerado de referencias del Annual Review of Energy, tomando como variable principal el idioma en que se encuentran escritos los documentos citados en los artículos publicados.

\section{Objetivos específicos:}

1. Conocer el nú me ro exac to de ar tícu los pu bli ca dos y de referencias, por volumen y año.

2. Iden ti fi car los idio mas de la li te ra tu ra ci ta da de las re fe rencias de los artículos.

3. Pre sen tar una dis tri bu ción del to tal de re fe ren cias, por año y según el idioma.

4. Determinar si las coautorías pre sen tan un fac tor cla ve para ele var el nú me ro y va rie dad de idio mas en la li te ra tu ra ci ta da.

5. De acuer do con la ins ti tu ción a la cual es tán vin cu la dos los autores, determinar cuántos y qué países han colaborado en la publicación de artículos.

6. Encontrar qué tópicos son los que inducen al científico a citar documentos escritos en idiomas extranjeros.

\section{METODO UTILIZADO}

En virtud de los pasos a seguir para efec tuar el pre sen te es tudio, los re sul ta dos ob te ni dos pue den sercon si de ra dos confiables y exactos.

Se ini ció con el plan tea mien to de las hi pó te sis, és tas orien taron la compilación de datos y establecieron relaciones significativas entre las diversas variables. Las hipótesis son:

1. Al re de dor de $190 \%$ del to tal de la li te ra tu ra ci ta da en los artículos científicos del Annual Review of Energy, (Vol. 1,
1976- Vol. 11, 1986) está escrita en la len gua a la cual per tenece la institución a la que están vinculados los autores.

2. Cerca del $95 \%$ de la literatura citada en los once volúmenes del Annual Review of Energy está escrita en inglés.

3. De los 190 ar tí cu los en es tu dio, más del $80 \%$ pre sen tan referencias en inglés.

4. Del número total de artículos, el índice de referencias en lenguas orientales no alcanza ni el 5 por ciento.

5. La literatura científica citada en los artículos de ciertos tópi cos que cu bren el es tu dio de un país o una par te de éste, está escrita en la lengua que se habla en esa región.

Una vez plan tea das las hi pó te sis, se ana li zó qué tipo de va riables se iban a considerar para la evaluación bibliométrica de ladocumentación citada en el Annual Re view of Energy. Los in di ca do res que se to ma ron en cuen ta fue ron los si guien tes:

1.- número de artículos publicados, según el volumen y el año;

2.- tópico que trata el artículo;

3.- número de autores por artículo;

4.- número de referencias;

5.- número de referencias, según el idioma;

6.- país al que pertene ce lains ti tu ción a la cual es tán vin cu lados los autores de los artículos.

Para la captación de datos se diseñó una forma especial, ésta permitió concentrar, en absoluto orden, la información de cada va riable. La téc ni ca bi blio mé tri ca uti li za da fue la ob servación directa de análisis de citas.

El ma te rial do cu men tal que se es tu dió abar có des de el Vol. 1, año 1976, has ta el Vol. 11, año 1986, con un to tal de 190 ar tículos y 10,211 referencias.

Los co men ta rios y re sul ta dos ob te ni dos se pre sen tan tan to en forma estadística (véase Anexo 1) como gráfica (véase Anexo 2).

\section{COMENTARIOS Y RESULTADOS}

Los da tos que nos brin da el cua dro 1 nos per mi ten apre ciar el número de artículos que se han publicado en cada uno de los volúmenes. El promedio de esta distribución es de 17.3 artículos por volumen.

En el cuadro 2 se puede observar el número de autores que presentan artículos en cada uno de los volúmenes. El promedio resultante de los once ejemplares analizados es de 28.2 autores por año. Los totales acumulativos de los cuadros 1 y 2 se pueden apreciar en la fig. 1 . 
El cuadro 3 presenta el número total de referencias que fueron ana li za das por vo lu men y año. De acuer do con el re sul tado general, el promedio de citas por volumen es de 928 referencias. La distribución del total acumulativo del cuadro 3 está en la fig. 2.

Los cuadros 1, 2 y 3 nos proporciona un panorama del universo documen tal es tu dia do para el pro pó si to de este trabajo. Los datos concentrados en el cuadro 4 nos permite manifestar lo siguiente:

1.- La colaboración de autores vinculados a instituciones no norteamericanas se presenta a partir del Vol. 2, año 1977.

2.- Los únicos países latinoamericanos que han publicado tra bajos en elAnnual Re view of Energy son Bra sil con dos artículos y México con uno.

3.- En cuanto a países orientales se refiere, Japón y Nueva Zelanda con un artículo.

4.- Los Estados Unidos, con un $83.2 \%$ mantiene un elevado número de artículos publicados en esta fuente.

5.- De los 17 paí ses que han dado a co no cer sus in ves ti ga ciones en el campo de la energía, Estados Unidos ocupa el primer lugar con un total de 150 artículos; siguiendo en orden descendente, Austria y Suecia y Canadá con 3; India, Japón, No rue ga, Bra sil y Fran cia con 2; Bél gi ca, Mé xi co, Is rael, Dinamarca, Italia y Nueva Zelanda con 1.

De los once volúmenes analizados, el $95.1 \%$ de la literatura citada está en inglés, el restante $4.9 \%$ se encuentra distribuido en los otros doce idio mas de tec ta dos. Esto se pue de ob servar en el cuadro 5. El ruso, considerado como el segundo idioma más importante para la transferencia de la información cien tífica, ape nas al can zó el in sig ni fi can te $1.05 \%$; elespañol, idioma oficial de casi toda América Latina, presentó so la men te el $0.35 \%$. La dis tri bu ción de ci tas se gún el idio ma se puede observar en la fig. 3 .

De 190 artículos publicados del Vol. 1, año 1976, al vol. 11, año 1986, sólo 36 presentaron referencias en más de un idioma (véa se cua dro 6). Los vo lú me nes co rres pon dien tes al año 1980 y 1981 se caracterizan por presentar artículos con referencias únicamente en inglés. Esto significa que más del $80 \%$, del to tal de ar tícu los pu bli ca dos en los once volú me nes, presentan literatura citada en un idioma y éste corresponde a la len gua que se ha bla en el país don de se en cuen tra la ins ti tución a la cual están vinculados los autores de los escritos.

En la fig. 4 se puede comparar el número de artículos con citas en un solo idioma, con la cantidad de artículos que presentan referencias en más de un idioma. Dicha figura muestra que los artículos que incluyen literatura citada en más de un idioma están muy por debajo con relación al gru po de documentos con citas en un solo idioma. Solamente el Vol. 2, año 1977, alcanzó la mitad de artículos con citas en más de un idioma.

En el cuadro 7 se aprecia que el $51.5 \%$ de los artículos han sido ela bo ra dos en for ma in di vi dual y el $48.5 \%$ co rres pon den a coautorías, con la participación de dos y hasta cuatro autores. De acuerdo al análisis de la documentación citada en los once volúmenes del Annual Review of Energy, podemos seña lar que las coau to rías no in flu yen en el he cho de ci tar obras en lenguas extranjeras. Es decir, tanto los artículos individuales como los elaborados por dos o más autores presentan referencias, en contadas ocasiones, en más de un idioma. La fig. 5 nos permite tener un panorama más objetivo sobre la distribución del número de estudios individuales y coautorías, según el año del volumen.

Cabe agregar que los artículos que tratan asuntos sobre un país en especial, son los que contienen referencias en idiomas distintos al inglés. Acorde con este resultado, se puede afirmar que los investigadores tienden a citar documentos principalmente en su propio idioma.

Tópicos que cubren los artículos que contienen referencias en más de un idioma.:

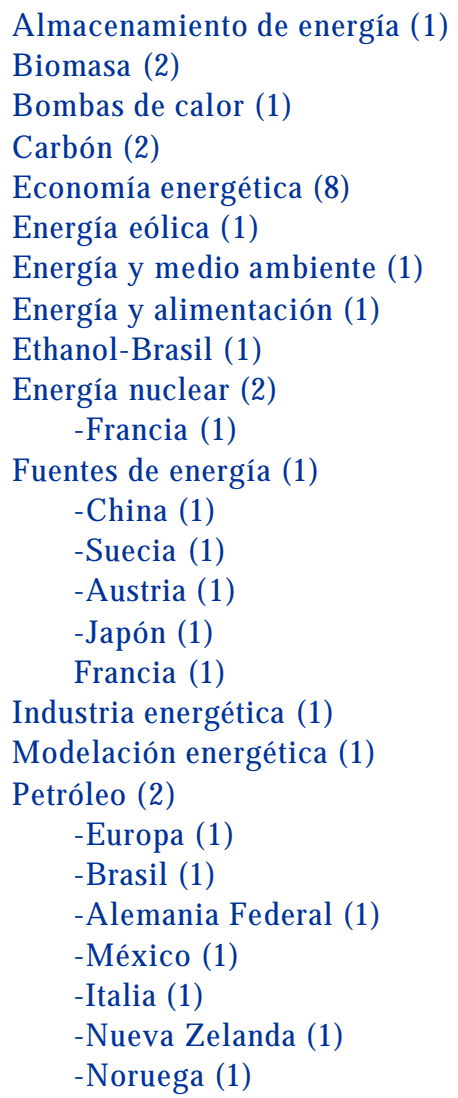

Nota: El número que se halla entre paréntesis corresponde a la fre cuen cia de ar tí cu los con ci tas en más de un idio ma so bre el tema correspondiente. 


\section{CONCLUSIONES}

Del estudio sobre el análisis idiomático de citas, del Annual Review of Energy, se derivan las siguientes conclusiones:

-El porcentaje de referencias en ruso, idioma calificado por varios ex per tos como la se gun da len gua más im por tan te para la transmisión del conocimiento científico, refleja la dificultad que presenta la barrera del idioma entre los autores que publican en la fuente antes dicha.

-La mayor parte de los estudiosos de paísesoccidentalesque han publicado en el Annual Review of Energy, entre los que sobresalen los norteamericanos, no citan literatura científica de pueblos eslavos y árabes, entre otros. Todo hace indicar que, por la dificultad que presenta la barrera del idioma, aquéllos tienden a citar obras principalmente en lengua materna.

-Los resultados obtenidos en este estudio son una muestra que per mi te co men tar que aún se está le jos de al can zar sa tis- factoriamente una mayor transferencia del conocimiento científico oriental en el área energética.

-El reducido porcentaje de citas sobre literatura científica orien tal per mi te afir mar que los es tu dio sos que pu bli can en el An nual Re view of Energy, no se in te re san por los ser vi cios de traducción que se ofrecen a nivel nacional e internacional, o ignoran la existencia de éstos.

-Los resultados que han arrojado las investigaciones, hechas por los autores que se citan en este trabajo sobre la barrera lin güís ti ca, se ase me jan a los ob te ni dos en esta in ves ti ga ción.

-Los países orientales y latinoamericanos presentan una reducida participación en la publicación de sus escritos en la fuente estudiada. Como consecuencia, existe una limitada oportunidad para conocer investigaciones del mundo oriental y de América Latina en el Annual Review of Energy.

-El número de artículos publicados, en el Annual Review of Energy, por los Estados Unidos deNortea mérica, al can zóun $83.2 \%$. De esta manera, este país sustenta el liderato.

\section{REFERENCIAS}

1. BER GEIJK, D. V. "Labarre ra lin güís ti ca en la di se mi na ción de la in for ma ción cien tífica”. Rev. Esp. Doc. Cient. Vol. 4, no. 3, (1980), p. 287-295.

2. MANZER, B. M. The abstract journal : 1720-1920. Metuchen, N. J. : Scarecrow Press, 1977, p. $215-228$.

3. PAUL, M. Periodicals administration in libraries. London Clive Bingley, 1978, p. 79.

4. PIGANIOL,P. "Cienciae in for mación: es tu dio pros pectivo" ConferenciaIntergubernamental para el Establecimientode un Sistema Mundial de Información Científica. Informe final. París : Unesco, 1971, p. 30-35.

5. PRICE, D. J. de Solla. Hacia una ciencia de la ciencia. Barcelona : Ariel, 1973, p. 33-106.

6. DAVIES, J. "Linguistic and politicalbarriers in the in ter na tio nal trans fer of in for ma tion in scien ce and te chno logy : a re interpretation". Journal of Documentation. Vol. 6 (1983), p. 171-181.

7. WOOD, D.N. "The fo reign lan gua ge pro blem fa cing scien tis tis and te chno lo gists in the Uni ted King tom : re port of a re cent survey". Journal of Documentation. Vol. 23, no. 2 (1967), p. 117- 131.

8. ANDERSON, J. D. "Foreign language barriers in information transfer". Journal of Education for Librarianship. Vol. 14, no. 3 (1984), p. 171-184.

9. KUNICKI, M. "La barriere linguistitique ; son importance et son evaluation”. Documentaliste. Vol. 17, no. 4-5 (juil-oct. 1980), p. 147-150.

10. DICKSON, A. J. "Librarians and the language barrier”. Aslib Proceedings. Vol. 31, no. 11 (nov. 1979$)$, p. $448-494$.

11. "El esperanto interlenguaje vivo de perspectiva universitaria". Gaceta UNAM. (15-ene.-1987), p. 16. 
ROWLEY, J.E. and Turner C. "Ba rrier to disse mi nation”.The disseminationofinformation. Boul der : Col. Westview Press, 1978 , p. 298-317.

13. NAGAO, M. "La traducción automática”. Mundo Científico. Vol. 4, no. 33 (1984), p. 298-317.

14. NOWAK, D. "The Na tio nal Trans la tions Cen ter : its de ve lopment, sco pe of ope ra tion and plans for the fu tu re" Scien ce and Technology. Vol. 2 (1985), p. 13-19.

15. AR NAIZ,E. "Laba rre ra del idio ma en la in for ma ción cien tífi ca y tec no ló gi ca y al gunos me dios para su pe rar la".Anua rio de Bibliotecología, (1983), p. 81-128.

16. PRUETT, J. “Trans la tions”. Scien tific and te chnicallibra ries: spe cialformats and subjetareas. Orlando, Florida : Academic Press, 1986. Vol. 2, p. 89-93.

17. AnnualReview Inc. : anonprofitscientific publisher; prospectus 1989. Palo Alto, Ca li for nia; An nual Re view Inc., 1988.48 p.

18. Annual Review of Energy 1987. Palo Alto, California : Annual Review Inc. Vol. 12.

\section{ANEXO 1 CUADROS}

\section{Cuadro 1}

Artículos publicados, según el volumen y el año

\begin{tabular}{|c|c|c|c|}
\hline VOL. & AÑO & NO. DE ARTICULOS & FRECUENCIA ACUMULADA \\
\hline 1 & 1976 & 27 & 27 \\
\hline 2 & 1977 & 18 & 45 \\
\hline 3 & 1978 & 13 & 58 \\
\hline 4 & 1979 & 14 & 72 \\
\hline 5 & 1980 & 12 & 84 \\
\hline 6 & 1981 & 16 & 100 \\
\hline 7 & 1982 & 13 & 113 \\
\hline 8 & 1983 & 16 & 129 \\
\hline 9 & 1984 & 20 & 149 \\
\hline 10 & 1985 & 21 & 170 \\
\hline 11 & 1986 & 20 & 190 \\
\hline
\end{tabular}




\begin{tabular}{|c|c|c|c|}
\hline \multicolumn{3}{|c|}{ Distribución de autores, por volumen y aito } & \\
\hline VOL. & AÑO & NO. DE AUTORES & FRECUENCIA ACUMULADA \\
\hline 1 & 1976 & 49 & 49 \\
\hline 2 & 1977 & 26 & 75 \\
\hline 3 & 1978 & 22 & 97 \\
\hline 4 & 1979 & 21 & 118 \\
\hline 5 & 1980 & 23 & 141 \\
\hline 6 & 1981 & 25 & 166 \\
\hline 7 & 1982 & 24 & 190 \\
\hline 8 & 1983 & 27 & 217 \\
\hline 9 & 1984 & 24 & 241 \\
\hline 10 & 1985 & 41 & 282 \\
\hline 11 & 1986 & 29 & 311 \\
\hline
\end{tabular}

\begin{tabular}{|cccc|}
\hline \multicolumn{5}{c|}{ Cuadro 3 } \\
Total de referencias, seguin el volumen y el año \\
\hline VOL. & NOÑ & NO. DE REFERENCLAS & FRECUENCLA ACUMULADA \\
\hline 1 & 1976 & 1361 & 1361 \\
2 & 1977 & 573 & 1934 \\
3 & 1978 & 1364 & 3298 \\
4 & 1979 & 852 & 4150 \\
5 & 1980 & 964 & 5114 \\
6 & 1981 & 895 & 6009 \\
7 & 1982 & 793 & 6802 \\
8 & 1983 & 786 & 7588 \\
9 & 1984 & 922 & 8510 \\
10 & 1985 & 1000 & 9512 \\
11 & 1986 & 699 & 10211 \\
\hline
\end{tabular}




\section{Cuadro 4}

Distribución de anticulos acordes al pais al que pertenece la institución a la cual están vinculados los autores, segün el año del voiumen.

\section{ORIGEN}

\begin{tabular}{|c|c|c|c|c|c|c|c|c|c|c|c|c|c|}
\hline AN̂̃O & 76 & 77 & 78 & 79 & 80 & 81 & 82 & 83 & 84 & 85 & 86 & TOTAL & $\%$ \\
\hline $\begin{array}{l}\text { ALEMANIA } \\
\text { OCCIDENTAL }\end{array}$ & - & 1 & - & - & - & - & 1 & - & 1 & - & - & 3 & 1.5 \\
\hline AUSTRIA & - & 3 & - & - & - & - & - & - & - & - & 2 & 5 & 26 \\
\hline BELGICA & - & 1 & - & - & - & - & - & - & - & - & - & 1 & 0.5 \\
\hline BAASHL & - & - & - & - & - & - & 1 & - & - & 1 & - & 2 & 1.2 \\
\hline CANLADA & - & 1 & - & 1 & - & - & - & 1 & - & - & - & 3 & 1.5 \\
\hline DINAMAACA & - & - & - & - & - & - & - & - & 1 & - & - & 1 & 0.5 \\
\hline Eua & 27 & 8 & 12 & 11 & 12 & 16 & 10 & 11 & 15 & 22 & 15 & 169 & 83.2 \\
\hline FFANCIA & - & - & - & - & - & - & - & 1 & 1 & - & - & 2 & 1.2 \\
\hline INDIA & 1 & - & - & - & - & - & - & - & - & 1 & - & 2 & 1.2 \\
\hline INGLATERRA & - & - & 1 & 2 & - & - & - & - & - & - & 1 & 4 & 2.1 \\
\hline TALA & - & - & - & - & - & - & - & - & 1 & - & - & 1 & 0.5 \\
\hline ISPAEL. & - & - & $*$ & - & - & - & - & 1 & - & - & - & 1 & 0.5 \\
\hline JAPON & - & 1 & - & - & - & - & 1 & - & - & - & - & 2 & 1.2 \\
\hline MEDCO & - & - & - & - & - & - & - & 1 & - & - & - & 1 & 0.5 \\
\hline NOFUEGA & - & - & - & 1 & - & - & - & - & - & - & 1 & 2 & 1.2 \\
\hline N. ZELANOA & - & - & - & - & - & - & - & - & 1 & - & - & 1 & 0.5 \\
\hline SUECA & - & 2 & - & - & - & - & - & 1 & - & 1 & 1 & 5 & 2.6 \\
\hline
\end{tabular}




\section{Cuadro 5}

Cantidad total de referencias por año, segîn el idioma

IDIOMAS

\begin{tabular}{|c|c|c|c|c|c|c|c|c|c|c|c|c|}
\hline ANO & 76 & $\pi$ & 78 & 9 & 80 & 81 & 82 & 83 & 84 & 85 & 86 & TOTAL \\
\hline INGLES & 1351 & 418 & 1357 & 35: & 964 & ass & 658 & 729 & 900 & 918 & 671 & 0712 \\
\hline Fuso & 1 & 104 & - & - & - & - & - & - & - & a & - & 106 \\
\hline FRANCES & - & 3 & 2 & - & - & - & - & 26 & B & B & - & 47 \\
\hline ESPANOL & - & 1 & - & - & - & - & - & 13 & - & - & 24 & 38 \\
\hline ALEMAN & 7 & 16 & 1 & - & - & - & 88 & 1 & - & 12 & 3 & 128 \\
\hline PORTUGUES & - & 1 & - & - & - & - & 24 & - & - & 27 & - & 52 \\
\hline ITALANO & - & - & - & - & - & - & - & - & 13 & 6 & - & 19 \\
\hline JAPONES & - & - & - & 1 & - & - & 23 & - & - & - & - & 24 \\
\hline SUECO & 2 & 30 & 4 & - & - & - & - & - & - & 21 & - & 57 \\
\hline DANES & - & - & - & - & - & - & - & - & 1 & 3 & - & 4 \\
\hline NOAUEGO & - & - & - & - & - & - & - & - & - & 3 & 1 & 4 \\
\hline HOLANOES & - & - & - & - & - & - & - & - & - & 1 & - & 1 \\
\hline HEBAEO & - & - & - & - & - & - & - & 17 & - & - & - & 17 \\
\hline TOTAL & 1361 & 573 & 1304 & 258 & 964 & eas & 780 & 786 & 922 & 1002 & 699 & 10211 \\
\hline
\end{tabular}

\section{Cuadro 6}

Articulos que presentan referencias en uno y más idiomas, según el año del volumen.

\begin{tabular}{|ccccc|}
\hline AÑO & $\begin{array}{c}\text { ARTICULOS CON } \\
\text { CITAS EN UN IDIOMA }\end{array}$ & $\%$ & $\begin{array}{c}\text { ARTICULOS CON CITAS EN } \\
\text { MAS DE UN IDIOMA }\end{array}$ & $\%$ \\
1976 & 25 & 92.5 & 2 & 75 \\
1977 & 9 & 56.0 & 9 & 50.0 \\
1978 & 9 & 69.2 & 4 & 30.8 \\
1979 & 13 & 92.9 & 1 & 7.1 \\
1980 & 12 & 100.0 & - & - \\
1981 & 16 & 100.0 &. & 23.1 \\
1982 & 10 & 76.9 & 3 & 18.7 \\
1983 & 13 & 81.3 & 3 & 25.0 \\
1984 & 15 & 75.0 & 5 & 28.5 \\
1985 & 15 & 71.5 & 6 & 15.0 \\
1986 & 17 & 85.0 & 3 & 18.95 \\
TOTAL & 154 & 81.05 & 36 & \\
\hline
\end{tabular}




\begin{tabular}{|c|c|c|c|c|c|}
\hline \multicolumn{6}{|c|}{ Distribución de estudios individuales y coautorias, segin el volumen y año } \\
\hline VOL & AN̂̀ & $\begin{array}{l}\text { ARTICULOS } \\
\text { INDIVIDUALES }\end{array}$ & $\%$ & $\begin{array}{l}\text { ARTICULOS } \\
\text { COAUTORIALES }\end{array}$ & $\%$ \\
\hline 1 & 1976 & 11 & 40.7 & 16 & $\$ 9.3$ \\
\hline 2 & 1977 & 11 & 61.1 & 7 & 38,9 \\
\hline 3 & 1978 & 7 & 53.9 & 6 & 46.1 \\
\hline 4 & 1979 & 7 & 50.0 & 7 & 50.0 \\
\hline 5 & 1980 & 4 & 33.3 & 8 & 66.7 \\
\hline 6 & 1981 & 9 & 56.2 & 7 & 43.8 \\
\hline 7 & 1982 & 4 & 30.8 & 9 & 69,2 \\
\hline 8 & 1983 & 9 & 56.2 & 7 & 43.8 \\
\hline 9 & 1984 & 17 & 85.0 & 3 & 15,0 \\
\hline 10 & 1985 & 7 & 33.3 & 14 & 66.7 \\
\hline 11 & 1986 & 12 & 60.0 & 8 & 40.0 \\
\hline TUTAL & & 98 & 51.5 & 92 & 48.5 \\
\hline
\end{tabular}

ANEXO 2 GRAFICAS

Fig. 1 Totales acumulativos de artículos y autores, según el año

NUMERO DE ARTICULOS Y AUTORES

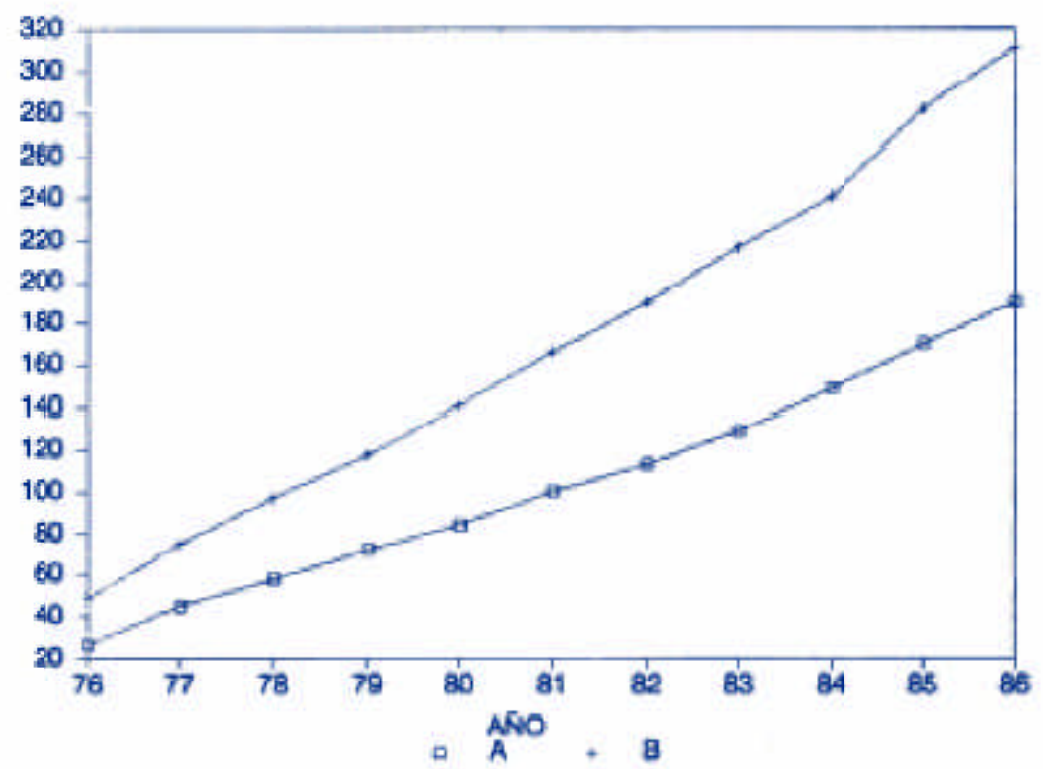

A: Total acumulativo de artículos publicados, segun el volumen.

B: Total acumulativo de autores, según el año del volumen. 
Fig. 2 Total acumulativo de referencias del Annual Review of Energy, según el año del volumen

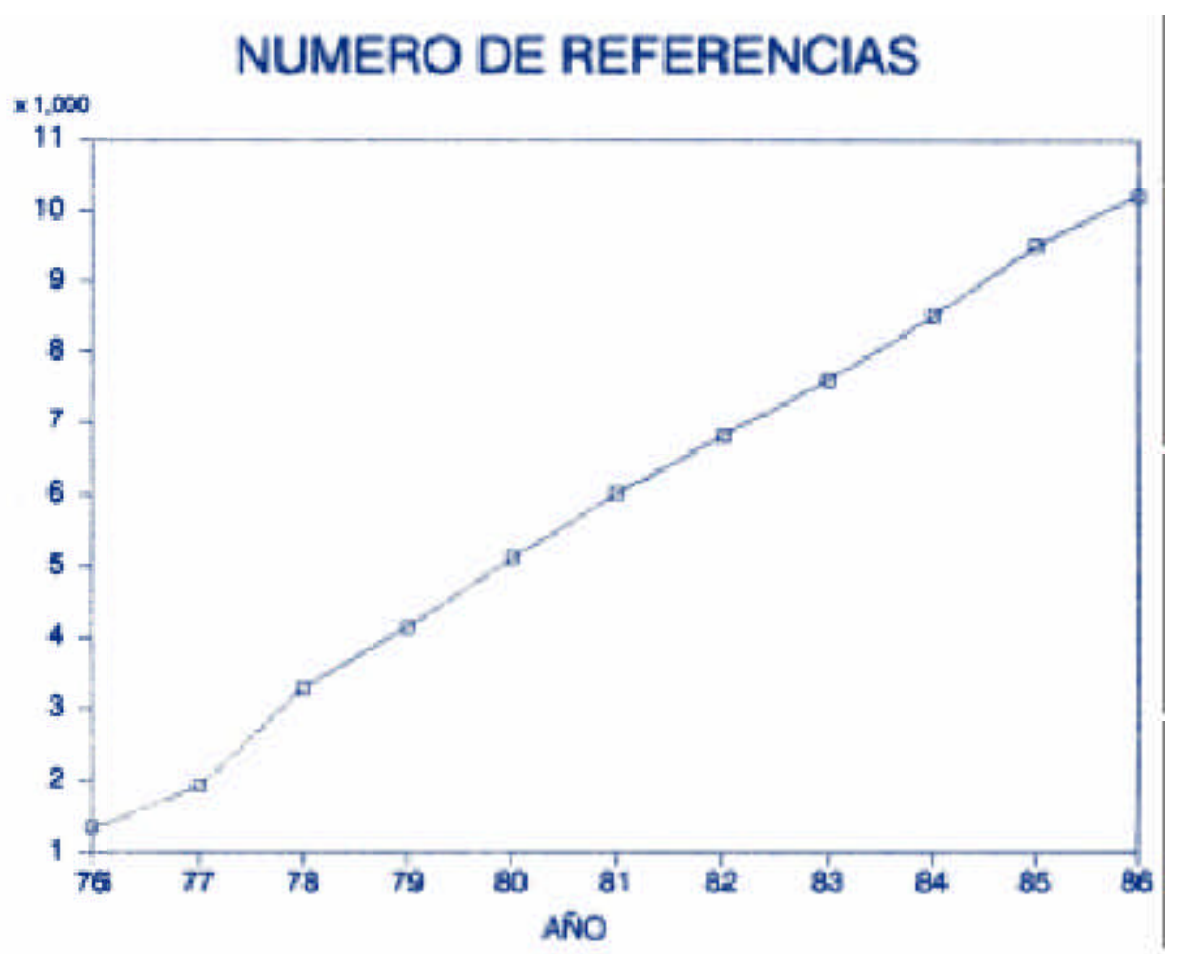

Fig. 3 Distribución de la literatura citada, según el idioma

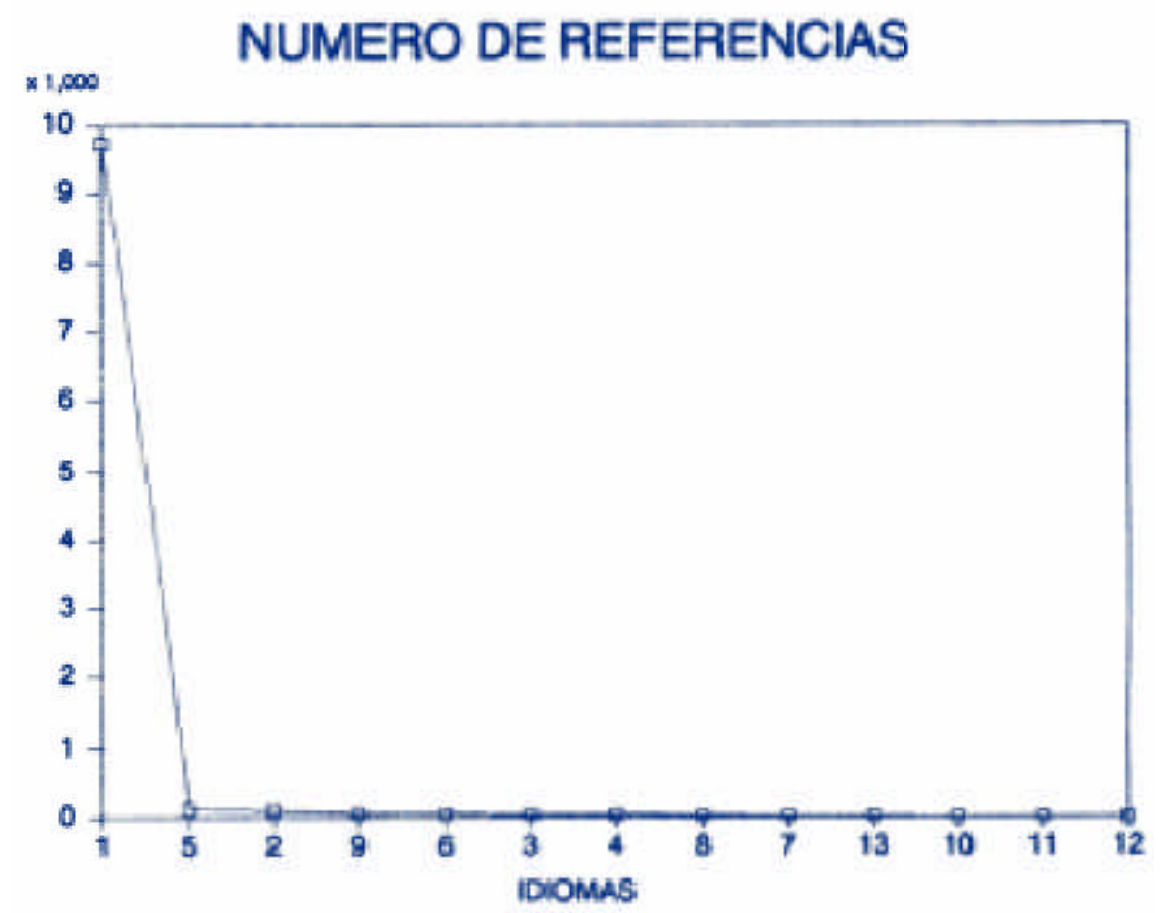

Idiomas:

$\begin{array}{llll}1 \text { Inglés 5 Alemán } & 9 & \text { Sueco } & 13 \text { Hebreo } \\ 2 \text { Ruso } & 6 \text { Portugués 10 } & \text { Danés } & \\ 3 \text { Francés } & 7 \text { Italiano } & \text { 11 Noruego } & \\ \text { 4 Español } & \text { 8 Japonés } & \text { 12 Holandés } & \end{array}$


Fig. 4 Distribución de artículos con literatura citada en uno y más de un idioma, según el año del volumen

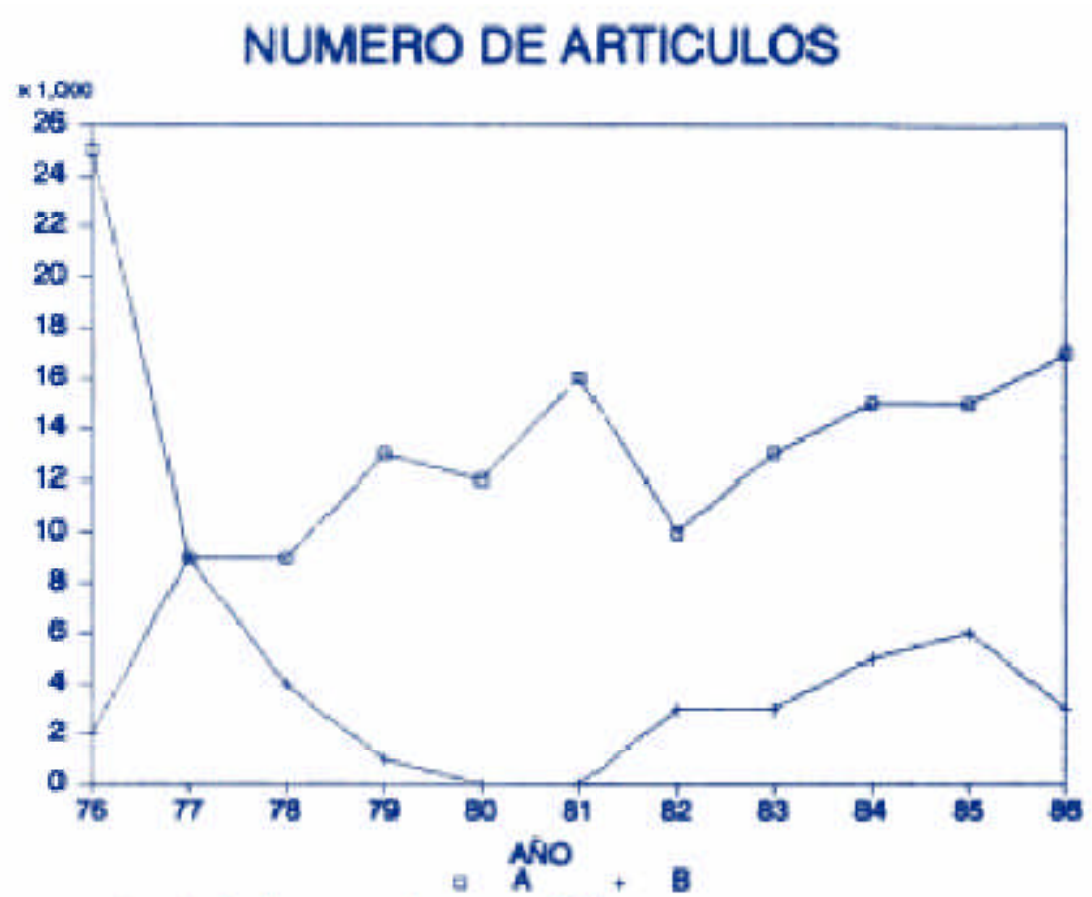

A: Articulos con citas en un idioma

B: Artículos con citas en más de un idioma

Fig. 5 Distribución de estudios individuales y coautorías, según el año del volumen

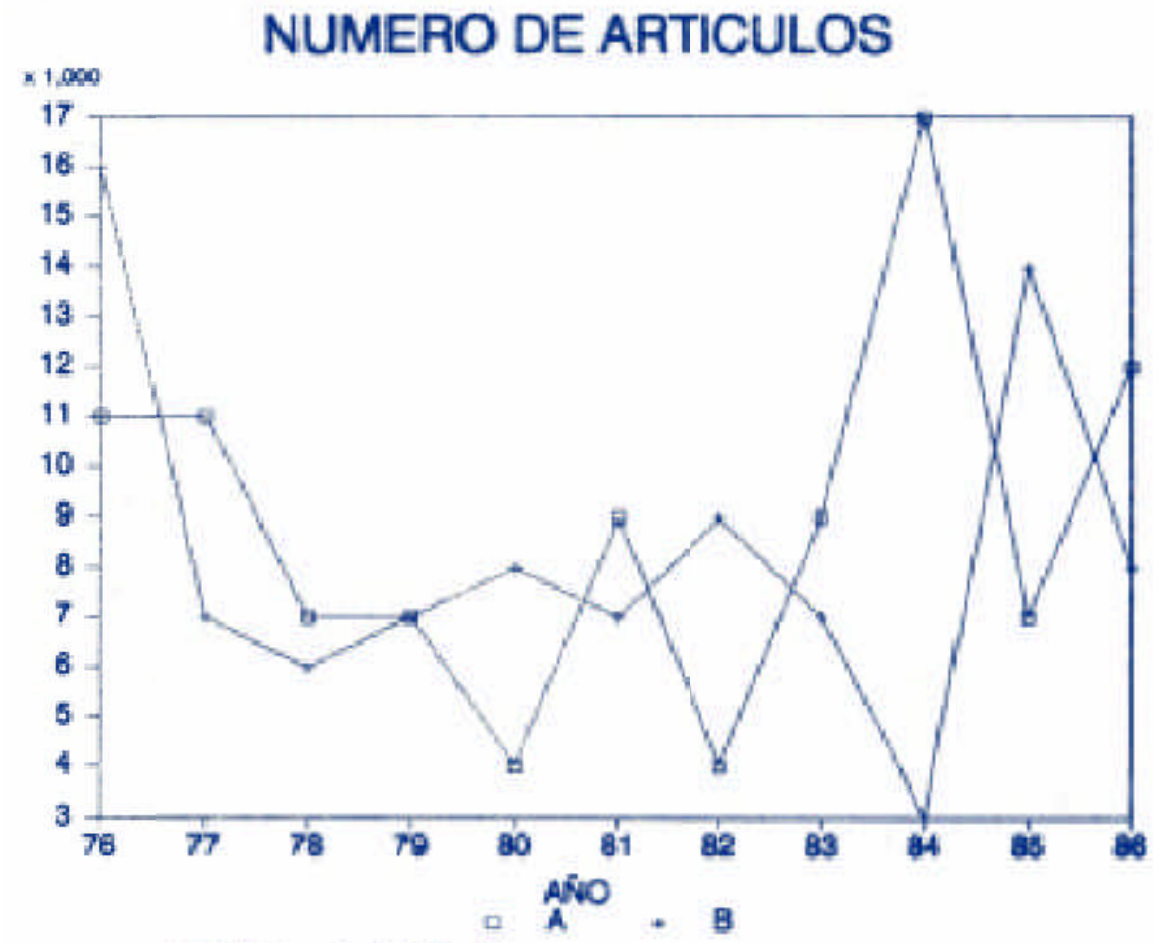

A: Trabajos individuales

B: Coautorias 